\title{
Impact of RGD Peptide Tethering to IL24/mda-7 (Melanoma Differentiation Associated Gene-7) on Apoptosis Induction in Hepatocellular Carcinoma Cells
}

\author{
Samaneh Bina ${ }^{1}$, Fatemeh Shenavar ${ }^{1}$, Mahboobeh Khodadad ${ }^{1}$, Mohammad \\ Reza Haghshenas ${ }^{2}$, Mojtaba Mortazavi ${ }^{3}$, Mohammad-Reza Fattahi ${ }^{1}$, Nasrollah \\ Erfani $^{2 *}$, Seyed Younes Hosseini ${ }^{1 *}$
}

Abstract

Background: Melanoma differentiation-associated gene-7 (MDA-7)/interleukin-24 (IL-24), a unique tumor suppressor gene, has killing activity in a broad spectrum of cancer cells. Herein, plasmids producing mda-7 proteins fused to different RGD peptides (full RGD4C and shortened RGD, tRGD) were evaluated for apoptosis induction with a hepatocellular carcinoma cell line, Hep-G2. The study aim was to improve the apoptosis potency of mda-7 by tethering to RGD peptides. Materials and Methods: Three plasmids including mda-7, mda-7-RGD and mda-7-tRGD genes beside a control vector were transfected into Hep-G2 cells. After 72 hours incubation, cell viability was evaluated by MTT assay. In addition, the rate of apoptosis was analyzed by flow cytometry using PI/annexin staining. To detect early events in apoptosis, 18 hours after transfection, expression of the BAX gene was quantified by real time PCR. Modeling of proteins was also performed to extrapolate possible consequences of RGD modification on their structures and subsequent attachment to receptors. Results and Conclusions: In MTT assays, while all mda-7 forms showed measurable inhibition of proliferation, unmodified mda-7 protein exhibited most significant effect compared to control plasmid $(\mathbf{P}<0.001)$. Again, flow cytometry analysis showed a significant apoptosis induction by simple mda-7 gene but not for those RGD-fused mda-7 proteins. These findings were also supported by expression analysis of $\mathrm{BAX}$ gene $(\mathrm{P}<0.001)$. Protein modelling analysis revealed that tethering RGD at the end of IL-24/Mda7 disrupt attachment to cognate receptor, IL-20R1/ IL-20R2. In conclusion, fusion of RGD4C and shortened RGD peptides to carboxyl terminal of mda7, not only reduce apoptosis property in vitro but also disrupt receptor attachment as demonstrated by protein modelling.

Keywords: mda-7/IL-24 - RGD peptide - apoptosis - HCC

Asian Pac J Cancer Prev, 16 (14), 6073-6080

\section{Introduction}

As a significant anti-cancer agent in the 21 st century, melanoma differentiation associated gene-7 (mda-7) /IL24 remained as a hopeful candidate for prospective cancer gene therapy (Yamamoto and Curiel, 2005). Mda-7 is a tumor suppressor gene which was first identified by using a subtraction hybridization approach from cDNA libraries of human melanoma cells (Huang et al., 2001; Caudell et al., 2002). The mda-7/IL-24 mRNA encodes a $23.8 \mathrm{kDa}$ polypeptide (Jiang et al., 1995) which after endogenous production, ultimately triggers apoptosis mainly through intracellular pathways including ER stress (Madireddi et al., 2002), overexpression of GADD genes (Sarkar et al., 2002), promoted mitochondrial dysfunction (Lebedeva et al., 2003), up-regulation of pro-apoptotic proteins and down-regulation of anti-apoptotic proteins (Sauane et al.,
2003). Based on some pioneer studies, supra-physiological levels of endogenous mda-7 protein induce apoptosis only in cancer cells, whereas no suppression of growth is observed in normal cells (Jiang et al., 1996; Fisher et al., 2003), indicating the natural tumor specific killing, as main goal of cancer gene therapy (Su et al., 2005).

Bystander effect that is a consequence of secreted protein binding/entering to nearby cells, is an established feature of mda-7 which improves its anti-tumor function, following production and distribution to the surrounding cells (Chada et al., 2004). Recently, the extracellular dependent ability of apoptosis induction by mda-7 has been more highlighted, which in turn emphasizes the role of bystander effect for this agent. Binding of mda-7/IL-24 to its cognate receptors (Kang D-c et al. 2003) activates signal transduction pathways mediating antitumor activity that is related to activation of BAX gene (Su et al., 2003;

${ }^{1}$ Gastroenterohepatology Research Center (GEHRC), ${ }^{2}$ Cancer Immunology Research Group, Shiraz Institute for Cancer Research, School of Medicine, Shiraz University of Medical Sciences, Shiraz, ${ }^{3}$ Department of Biotechnology, Institute of Science and High Technology and Environmental Science, Graduate University of Advanced Technology, Kerman, Iran *For correspondence: Erfanin@sums.ac.ir,Hosseinisy22@yahoo.com, 
Lebedeva et al., 2007). Also, this binding improves halflife of mda-7 mRNA through uncharacterized mechanisms which finally lengthens the antitumor effect duration (Madireddi et al., 2000; Sarkar et al., 2007)

Finding an effective approach for driving therapeutic agents toward the site of tumor is among the most important aspects of gene therapy (Bazan-Peregrino et al., 2007) One of the accepted ways for this purpose, is targeting those cell surface proteins altered during tumor development, among which integrins $\alpha v \beta 3, \alpha 5 \beta 1$ and $\alpha v \beta 6$ could be beneficial(McLane et al., 1996; Chada et al., 2004). Due to the high affinity of RGD (Arg-Gly-Asp) peptide to integrin (Assa-Munt et al., 2001; Xiong et al., 2002) conjugation of mda-7/IL-24 with RGD sequence is supposed to make a concentrated dose of this apoptotic factor in tumor site, which consequently induces more apoptosis of the neighboring cells by bystander effect (Pei et al., 2012; Whitaker et al., 2012). While a dozen of experiments have demonstrated the potential role of RGD in improving both efficiency and targeting of anti-tumor agents, very limited data have been published about the fusion of mda-7 and RGD peptide.

On the basis of amino acid sequence similarity and common receptors, IL-19, IL-20, and IL-24 have been designated as IL-20 subfamily cytokines (Kunz et al., 2006; Logsdon et al., 2012). Therefore, available crystal structure of IL-20 can provide the basis for further three-dimensional (3D) molecular modeling of IL-24 (McGuffin et al., 2000). In the following, the protein structure modelling was employed to give a better description for outcomes of IL-24 protein modification and its consequences on protein interaction with IL-20R1/ IL-20R2 receptor.

The present study evaluated the apoptosis potency of 3 different constructs expressing standard mda-7 protein with or without RGD peptide at the end. This modification will improve specificity of mda-7 targeting new tumor vessels and cells (Fisher, 2005) Assa-Munt N et al., 2001) that in addition to inhibiting angiogenesis it may trigger apoptosis of tumor cells expressing integrin ligands as well (Chada et al., 2007; Lebedeva et al., 2007; Sarkar et al., 2007). To examine and consolidate this concept, first we assessed apoptosis induction in a HCC related cell, i.e. HepG2 line. Also, we tested the standard RGD cyclic form and a shortened RGD motif, as explained before (Hersel et al., 2003; Holig et al., 2004). As for a peptide sequence, the possible immunogenic role of standard RGD sequence is inevitable, the truncated form of RGD surely reduces this potential immunogenicity and consequently improves its safety (Marchand-Brynaert et al., 1999; Craig et al., 2008).

\section{Materials and Methods}

\section{Plasmids, Reagent, cell line and kits}

Three constructs including pmda-7, pmda-RGD and pmda-tRGD plasmids were prepared previously in our lab, as described in our previous study (Khodadad M, In press). All the plasmids contained an mda-7 sequence while two different tags tethered to pmda-RGD and pmdatRGD. The RGD tag included the standard cyclic RGD4C sequence while the tRGD contained truncated form of sequence, nominated as tRGD in this study. In addition to respective genes, all the constructs were also containing a GFP sequence which makes them traceable following transfection and during apoptotic detection. The empty p-AdenoVator-CMV5-IRES-GFP was also enrolled as the negative control that denominated as pIRES.

All cell culture agents and Lipofectamine 2000 transfection reagent were obtained from Invitrogen Company. MTT [3-(4, 5-dimethylthiazole-2-yl)-2, 5-diphenyl tetrazolium bromide] powder was provided from Sigma company, USA. HepG2 cells were prepared by national cell bank (Pasteur Institute of Iran). PI/ Annexin (APC conjugated) kit was from BD Company. The cDNA synthesis kit and Real time master mix reagent was purchased from Bioneer Company (S. Korea). Other molecular materials were purchased from Cinaclon Company (Iran).

\section{Cell culture and transfection}

The cells were grown in DMEM with $10 \%$ fetal bovine serum (FBS), 100u/ml Penicillin, streptomycin and 1\% L-Glutamax, then, maintained at $37^{\circ} \mathrm{C}, 5 \% \mathrm{CO} 2$. HepG2 cells $\left(2 \times 10^{5}\right.$ cells $)$ were seeded in 24 -well plate and after 24 hours in $80 \%$ confluence, they were transfected with $1 \mu \mathrm{g}$ DNA plasmid using Lipofectamine 2000 reagent in serum free DMEM medium, according to the manufacturer's instructions. About 7 hours after transfection, the medium was replaced with fresh complete DMEM medium and the plate was left up to 24 and 72 hours for mRNA expression and apoptosis analysis, respectively.

\section{MTT assay}

In order to check cell viability after transfection, MTT assay was performed for 3 consecutive times. Cells in logarithmic growth phase were cultured for 24 hours in 48 -well plates $\left(1 \times 10^{5}\right.$ cells per well). After transfection, cells were incubated for further 72 hours. Mitochondrial function, a sign of viability, was evaluated by the traditional colorimetric assay, MTT. Briefly, the medium was removed and a fresh medium containing $0.5 \mathrm{mg} / \mathrm{ml}$ MTT was added to each well. The cells were incubated at $37^{\circ} \mathrm{C}$ for 4 hours, afterwards, supernatant was removed while $50 \mu$ dimethylsulfoxide was replaced in each well and, then, cells were incubated for further 30 minutes at $37^{\circ} \mathrm{C}$ with gentle shaking. Finally, absorbance was read on a microplate reader at $570 \mathrm{~nm}$. Cell viability was calculated as the ratio of the absorbance of the test groups to that of the control group (untreated cells).

\section{Apoptosis assay by flow cytometry}

Transfected cells were harvested by the help of dissociation buffer 72 hours post transfection and introduced into apoptosis assessment process by APC Annexin-V Apoptosis Detection Kit (BD Inc. USA), according to the manufacturer's instructions. Briefly, cells were washed by PBS and re-suspended in Binding Buffer $1 \%(200 \mu \mathrm{L})$. The cells were then, aliquated and stained with Annexin-V conjugated APC and propidium iodide (PI) for 15 minutes in dark. Then, $300 \mu \mathrm{L}$ Binding Buffer was added and acquisition was immediately performed on a FACS Calibur flow cytometer (BD 
Biosciences). Analyses were done using Cell Quest Pro Software package. The sum of early and late apoptosis percentages was considered as total percentage of total apoptosis. In addition, during cell analysis, both GFP+ (received plasmids) and total population (both transfected and untransfected cells) were evaluated to investigate the role of secreted cytokine and subsequent bystander effect.

\section{Real-time PCR}

After 18 hours post transfection, total RNAs were extracted from the cells using RNA extraction kit, according to the provided protocol. Then, $1 \mu \mathrm{g}$ total cellular RNAs were reverse transcribed using a cDNA synthesis kit (Bioneer Inc.) after adding reverse primers.

Quantitative PCR was performed in final volume of $40 \mu \mathrm{L}$ by SYBR Green Master mix containing all PCR gradients, 10 pmol of each primer and $3 \mu 1 \mathrm{cDNA}$ template using the thermal cycler (BIO-Rad System). All samples quantification was performed in triplicates, and human glyceraldehyde-3-phosphate dehydrogenase (hGAPDH) enrolled also as a control gene in each PCR run. Relevant primer pair sequences employed here is shown in Table1. In PCR program, after an initial denaturation step of 60 seconds at $95^{\circ} \mathrm{C}$, conditions for cycling included 35 cycles of 10 seconds at $95^{\circ} \mathrm{C}$, and 35 seconds at $55^{\circ} \mathrm{C}$.

Relative expression of BAX genes was calculated after counting the difference between $\mathrm{Ct}$ of test group and housekeeping gene by 2 (Ct of GAPDH-Ct of Test) $\times 1000$ formula. Data were entered and analyzed in Excel program, then, reported as a crude change in mRNA levels.

\section{Recombinant IL-24/mda-7 modeling}

Construction of three-dimensional (3D) molecular modeling of wild-type IL-24 and recombinant IL-24/mda7 was conducted with I-TASSER server (Zhang, 2008). In this server, 3D models built based on multiple-threading alignments by LOMETS (Wu and Zhang, 2007), iterative template fragment assembly simulations and matching the $3 \mathrm{D}$ models with BioLiP protein function database. The positions of secondary structures of IL-24/mda7 protein in LOMEST were predicted by PSI-PRED(McGuffin et al., 2000) . The LOMETS generates 3D models by collecting high-scoring target-to-template alignments from 9 locally-installed threading programs (Wu and Zhang, 2007). Models that have the beast Z-score and "Confidence Score" were chosen by I-TASSER server. Regarding the same receptors for IL-24 and IL-20 attachments, to further understand IL-24 interaction with its receptor IL20R1/IL-20R2 ternary complex was constructed based on crystal structure of IL-20/IL-20R1/IL-20R2 complex as previously determined (Logsdon et al., 2012). For this, IL-20 (chain A)/IL-20R1 (chain B)/IL-20R2 (chain R) complex (4DOH.pdb) and IL-24 (model1.pdb) were loaded into PyMOL program (DeLano, 2002) and align them to get a model. For studying the situation of RGD peptide in ternary complex, the recombinant IL-24 was loaded into PyMOL program and aligns them to get a model. Hydrogen bonds of wild-type and recombinant IL24 proteins were also calculated by SWISS-PDB Viewer program (Schwede et al., 2003).

\section{Statistical analysis}

All the tests were performed at least three times and the results were expressed as mean \pm SD. Statistical difference between means were evaluated using one-way ANOVA test following Tukey post-test survey. Significance level was set at $\mathrm{P}<0.05$.

\section{Results}

\section{MTT Viability assays}

Once HepG2 cells were transfected by three test plasmids, their proliferation and viability were investigated by MTT assay, 72 hours following transfection. The results showed that among different mda-7 forms, anti-proliferative activity of normal mda-7 was more significantly higher when compared to GFP control group as depicted in Figure 1. The viability percentages of different groups were as follows: $46 \%(\mathrm{p} \leq 0.001)$ for pmda-7 receiving group, $57 \%(\mathrm{p} \leq 0.05)$ for pmda-7-RGD, $60 \%(\mathrm{p} \leq 0.05)$ for pmda-7-tRGD and $77 \%$ for pIRES.

\section{Evaluation of apoptosis by flow cytometry}

Transfected cells were harvested after 72 hours post transfection and submitted to flow cytometry to quantify GFP expression signals. The transfection rate of Hep-G2 cells measured $50-60 \%$, among different groups with no significant difference. Notably, all plasmids had built-in GFP gene which led to the emission of fluorescent light in FL1 channel in the cells which received the plasmids.

The Annexin-V/PI staining assay was employed to quantify the apoptosis effect of three constructed plasmids (pmda-7, pmda-7-RGD and pmda-7-tRGD plasmids) and compared with control IRES plasmid on Hep-G2 cells.

Besides assessment of apoptosis in the cells which received the constructed plasmids (GFP+ cells), and in order to simultaneously assess the bystander effect of mda-7 on the neighboring cells which did not receive the constructed plasmids (GFP- cells), two different analyses were performed on the flow cytometry raw data. In the first set of analyses (Figure 2 and Table 2), the percentages of Annexin and PI positive cells were calculated among

\section{Table 1. Primer Sequences Employed in the Study}

\begin{tabular}{llc}
\hline Primer & \multicolumn{1}{c}{ Sequence $(5 / \rightarrow 3 /)$} & $\begin{array}{c}\text { Fragment } \\
\text { Size }(\mathrm{bp})\end{array}$ \\
\hline hGAPDH & F: GAAGGTGAAGGTCGGAGTC & $226 \mathrm{bp}$ \\
& R:GAAGATGGTGATGGGATTC & \\
BAX & F: TGCTTCAGGGTTCATCCAG & $170 \mathrm{bp}$ \\
& R: GGCGGCAATCATCCTCTG & \\
\hline
\end{tabular}

Table 2. The Apoptosis rate of Different test Groups after Receiving Corresponding Plasmids

\begin{tabular}{lcc}
\hline & \multicolumn{2}{c}{ Total Apoptosis } \\
\hline plasmid & both GFP+ and GFP- cells & only GFP+ cells \\
\hline mda-7 & $52.47 \%(\mathrm{p}=0.05)$ & $37.64 \%(\mathrm{p}=0.05)$ \\
mda-7-RGD & $26.17 \%(\mathrm{P}=0.513)$ & $18.03 \%(\mathrm{p}=0.05)$ \\
mda-7-tRGD & $31.86 \%(\mathrm{p}=0.127)$ & $19.92 \%(\mathrm{p}=0.05)$ \\
IRES & $23.11 \%$ & $12.71 \%$ \\
\hline
\end{tabular}




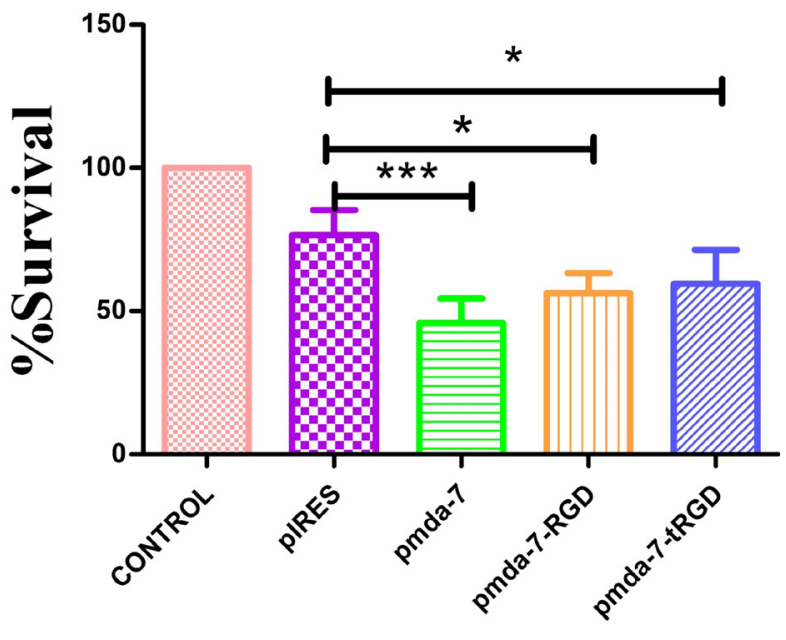

Figure 1. The results of survival rate among different groups. Cell groups were transfected by different plasmids, then, MTT assay performed to measure cell survival. The assay indicated that pmda-7 induces the most significant $(\mathrm{P}<0.001)$ decrease in survival (46\% viability), compared to the control group, pIRES (77\% Viability)
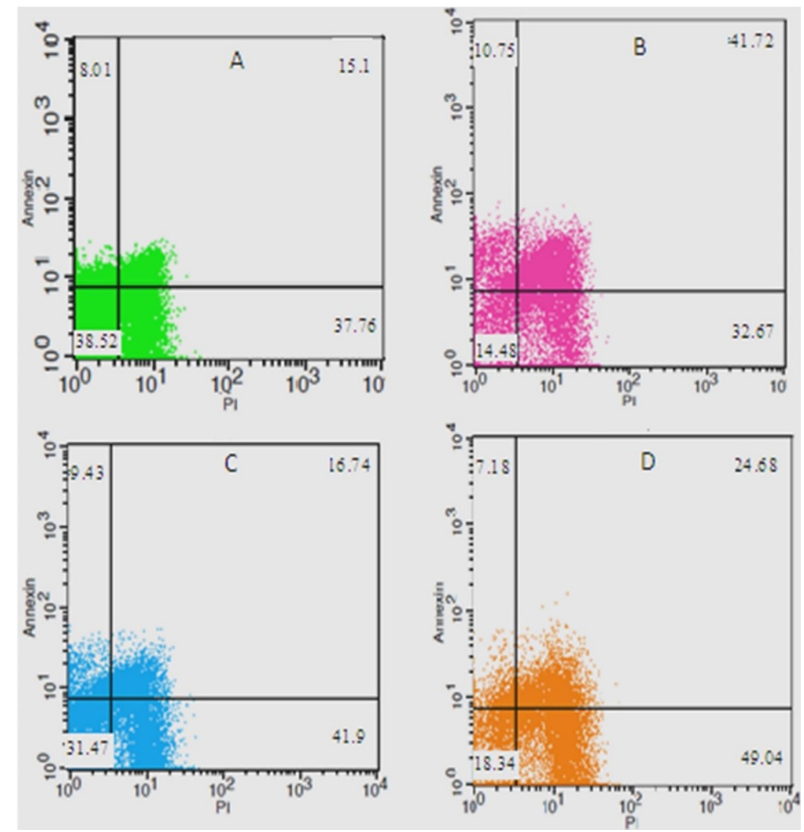

Figure 2. Flow Cytometry Analysis of Apoptosis/ Necrosis of the Samples Undergoing Transfection with the constructed plasmids (mda-7 (B), mda-7-RGD (C) and mda-7-t RGD (D), as well as IRES (A) control plasmid. The mean percentages \pm SD of the early (up left) and the late apoptosis (up right), as well as necrosis for each construct are tagged in the figure. The mean percentage $\pm \mathrm{SD}$ of the early (up left), the late (up right) and total apoptosis for mda-7 are, respectively $10.75 \%, 41.72 \%$ and $52.47 \%$, for mda-7-RGD, $9.43 \%, 16.74$ and $26.17 \%$, for mda-7tRGD $7.18 \%, 24.68 \%$ and $31.86 \%$, for IRES (control plasmid) $8.01 \%, 15.1 \%$ and $23.11 \%$. Total HepG2 cells (both GFP+ and GFP-) were included in final analysis

total acquired cells (both GFP+ and GFP- cells), and in the second set of analyses (figure 3 ), the percentages were calculated only in the cells that received the constructed
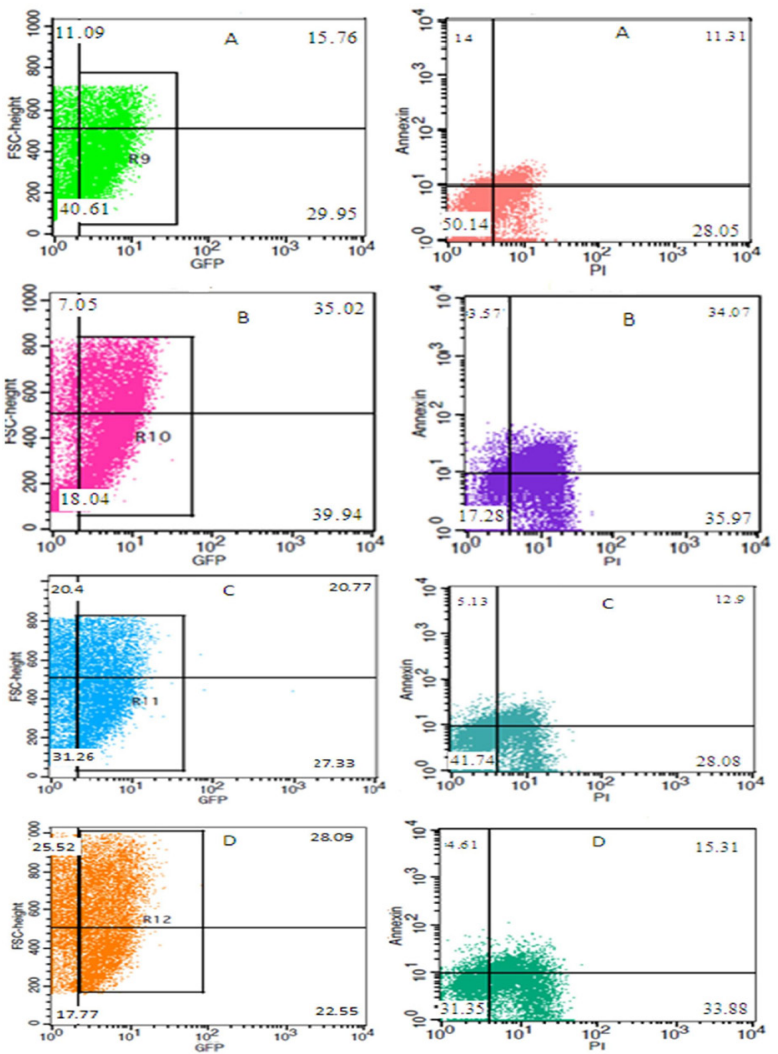

Figure 3. Flow Cytometry Analysis of Apoptosis/ Necrosis of HepG2 Cells Receiving Constructed Plasmids (pmda-7(B), pmda-7-RGD (C) and pmda-7tRGD (D) as well as pIRES (A) control plasmid. The mean percentage \pm SD of the early and the late apoptosis, as well as necrosis for each construct is tagged in the figure. The mean percentage $\pm \mathrm{SD}$ of the early (up left), the late (up right) total apoptosis for mda-7, are respectively $3.57,34.07$ and $37.64 \%$, for pmda-7-RGD, $5.13 \%, 12.9 \%$ and $18.03 \%$ and for pmda7-tRGD $4.61 \%, 15.31 \%$ and $19.92 \%$ and pIRES control are respectively $1.4 \%, 11.31 \%$ and $12.71 \%$. It should be noted that only GFP+ HepG 2 cells (those which received plasmids) were first gated separately, then, analyzed as cells received plasmids and included in final analysis

plasmids (only GFP+ cells).

As shown in figure 2, the percentage of total apoptosis (early and late stages) for total acquired cell population (both GFP+ and GFP- cells) $72 \mathrm{~h}$ after transfection were: $52.47 \%(\mathrm{p}=0.05)$ for mda- $7,26.17 \%(\mathrm{P}=0.513)$ for mda-7RGD, $31.86 \%(\mathrm{p}=0.127)$ for mda-7-tRGD and $23.11 \%$ for IRES. In comparison to total population analysis, similar results achieved for the second set of data. In this analysis, the percentage of apoptotic events among the cells that received the constructed plasmids (only GFP+ cells) were as follows: $37.64 \%(\mathrm{p}=0.05)$ for mda-7, $18.03 \%(\mathrm{p}=0.05)$ for mda-7-RGD, $19.92 \%(\mathrm{p}=0.05)$ for mda-7-tRGD and $12.71 \%$ for IRES.

In sum, flow cytometry analysis showed that pmda-7 plasmid exhibited a significant induction of apoptosis among both total and GFP+ cells $(\mathrm{P} \leq 0.001)$. The negligible differences between the rates of apoptosis in total and GFP+ population (52.47\% vs $37.64 \%$ ) indicated the possible role for bystander effect of this cytokine after secretion into cell culture as depicted in 


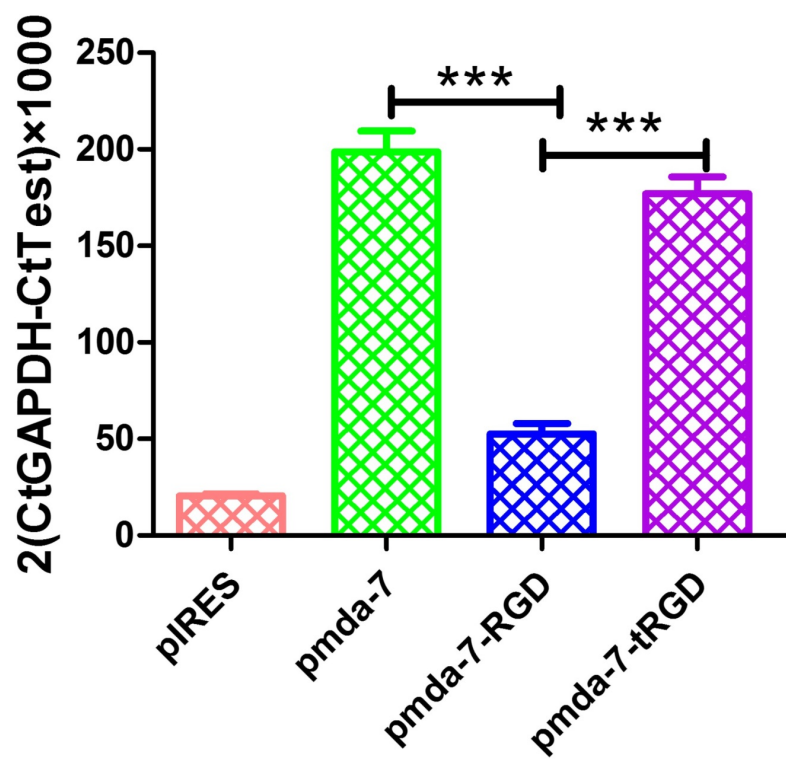

Figure 4. The mRNA expression analysis for BAX gene. Real-time PCR was performed after 18 hours post transfection to detect early apoptotic events. These results indicated that BAX expression significantly rose up for pmda-7 and pmda-7-tRGD, compared to pmda-7-RGD group $(\mathrm{P}<0.001)$. The data were represented as mean of at least $3 \mathrm{PCR}$ reactions \pm SD
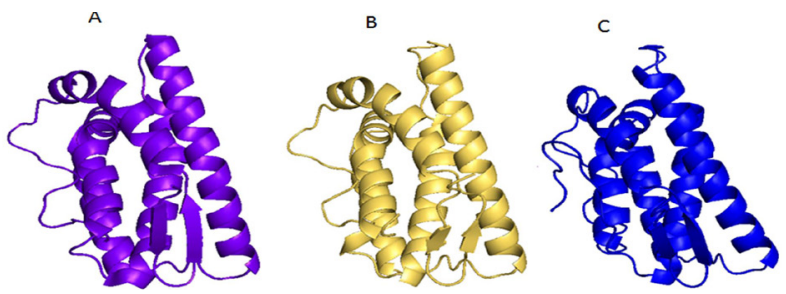

Figure 5. Crystal structure of IL-20 (PDB ID: 4DOH) (A) and molecular models of the wild-type IL-24 (B) and recombinant IL-24 (C) (based from IL-20). Cartoon representation of the IL-20, wild-type IL-24/mda7 and recombinant IL-24(RGD fused) illustrating the overall folds of these interleukin

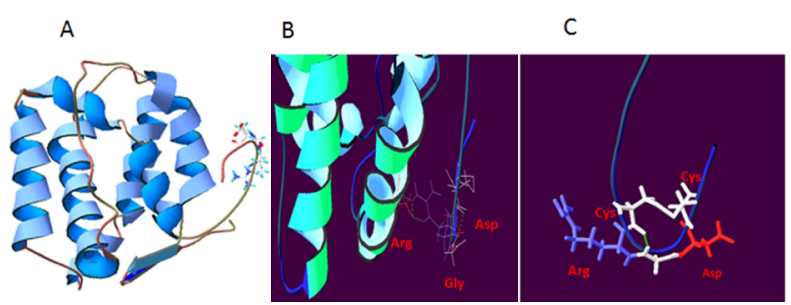

Figure 6. Stereo View of Recombinant Il-24 and Situation of RGD-4C Peptide. (A) Views of the Il-24 represent in carton with RGD motif regions represent in stik. (B) Views of the RGD motif regions that formed hydrogen bond with helix of recombinant IL-24. (C) View of RGD motif regions that formed disulfide bond

Table 2. Furthermore, the analysis indicated the increased percentage of apoptosis in the HepG2 cells receiving mda-7-RGD and mda-7-tRGD, compared to that in IRES, control plasmid, but not significantly different $(\mathrm{P}>0.05)$. Similarly, no significant difference was observed in the percentage of apoptosis between mda-7-RGD and mda7-tRGD in both sets of analysis, although a tendency toward higher apoptosis induction was detected in the cells receiving the former construct.
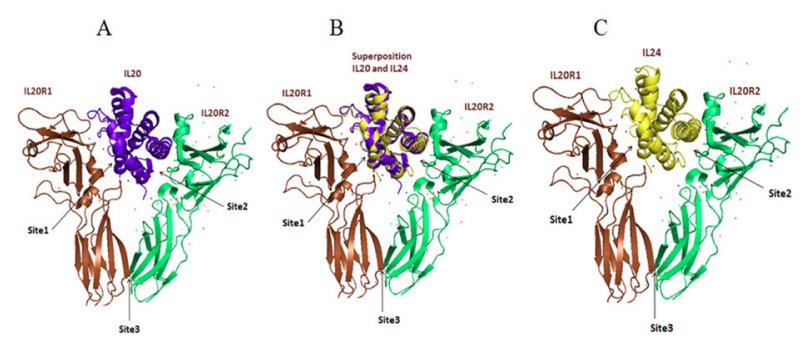

Figure 7. (A) Ribbon diagram of the IL-20 ternary signaling complex and three protein interfaces, IL-20/ IL-20R1 (site 1), IL-20/IL-20R2 (site 2), and IL-20R1/ IL-20R2 (site 3) (B) Superposition of the IL-20 (red), and IL-24 (yellow) in ternary complex. (C) Ribbon diagram of the IL-20 ternary signaling complex and three protein interfaces IL-24/IL-20R1 (site 1), IL-24/ IL-20R2 (site 2), and IL-20R1/IL-20R2 (site 3)
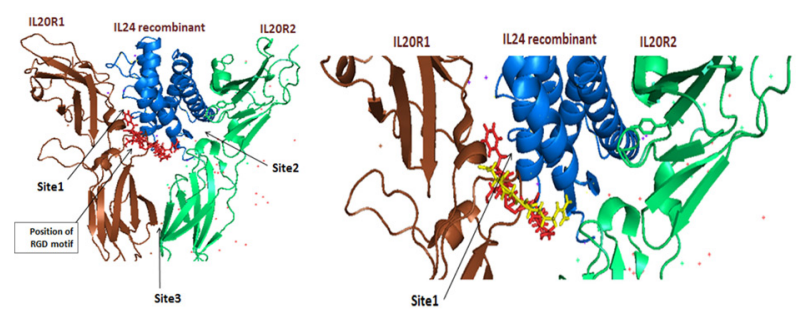

Figure 8. (A) Ribbon diagram of the IL-24rec ternary signaling complex (blue) and three protein interfaces IL-24/IL-20R1 (site 1), IL-24/IL-20R2 (site 2), and IL-20R1/IL-20R2 (site 3) and position of RGD motif (red) (B) big view from position of sequence that added (red light) and RGD motif (yellow) in c-terminal of IL24rec (blue) in ternary complex and protein interfaces IL-24rec/IL-20R1 (site 1)

\section{Real-Time PCR}

To determine the apoptosis-inducing effects of different plasmids expressing mda-7 in HepG2 cell, the mRNA levels of BAX as pro-apoptotic gene, were tested by real-time PCR. Based on the results for BAX expression, pmda-7 and pmda-7-tRGD showed a significant induction of this gene $(\mathrm{P} \leq 0.001)$ which is indicative of more apoptosis in cells (figure 4).

Modeling the normal and modified IL-24/Mda-7 proteins

A well-defined 3D structure of the wild-type IL-24/ mda7 was not resolved, so far. In order to predict the structures of wild-type and recombinant IL-24 with/ without RGD peptide in ternary complex, for first time we constructed 3D molecular models of the wild-type and recombinant IL-24 based on structure of IL-20 (PDB ID: 4DOH) (fig.1). As shown, the overall folding of wild-type and recombinant IL-24 and location of $\alpha$-helix and loops in these models have reliably predicted and occupied similar situation relative to IL-20 (fig. 5).

We also studied the situation of RGD peptide relative to IL-24/mda7. As shown in figure 6 the results of modeling demonstrated that both RGD peptide moieties formed a distinct surface loop in C-terminal of IL-24 with a disulfide bond. 
Samaneh Bina et al

The modeling of RGD peptide in protein-receptor interaction

As IL-24 and IL-20 share their receptors, this concept employed to predict the structure of IL-24/IL-20R1/ IL-20R2 ternary complex. For study the position of RGD peptide in interaction with IL-20R1 and IL-20R2 receptors, the IL-24rec/IL-20R1/IL-20R2 ternary complex was modeled. As previously mentioned, IL-20/IL-20R1/ IL-20R2 ternary complex formation is mediated by three protein interfaces, IL-20/IL-20R1 (site 1), IL-20/IL-20R2 (site 2), and IL-20R1/IL-20R2 (site 3). The IL-20/IL20R1 site 1 interface consists of two contact surfaces, site $1 \mathrm{a}$ and site $1 \mathrm{~b}$. Site 1a is formed by IL-20R1 L2-L4 loops that contact a small cavity on IL-20 located at the intersection of helix $\mathrm{F}$ and $\mathrm{AB}$ loop. Site $1 \mathrm{~b}$ contacts occur between IL-20R1 L6 and the N-terminus of helix A. As shown in figure 7 the IL-24 in the ternary complex occupied similar situation in comparison with IL-20 and three protein interfaces, IL-24/IL-20R1 (site 1), IL-24/IL-20R2 (site 2), and IL-20R 1/IL-20R2 (site 3) were formed.

As RGD peptide was introduced at the end of IL-24 structure, this motif buried in the center of the ternary complex and disrupted the non-covalent interactions that mediated the formation of ternary complex by IL-24/IL$20 \mathrm{R} 1$ (site 1). The overall effects of RGD peptide was to decreased the affinity of IL-24red to IL-20R1-IL-20R2 receptors and result in low effects in comparison with control.

\section{Discussion}

A huge part of cancer gene therapy approaches try to introduce apoptosis inducer genes for the elimination of tumor cells. The mda-7, as a cytokine is a tumor suppressor gene that induces apoptosis in a wide spectrum of cancer cells, following extraordinary endogenous production (Sauane et al., 2003; Gupta et al., 2006). The bystander effect of this secretory protein has been considered recently. It was demonstrated that binding of mda-7/IL24 to its cognate receptors activates signal transduction pathways mediating antitumor activity that is related to the activation of BAX gene (Su et al., 2003; Lebedeva et al., 2007)and increasing the half-life of mda-7 mRNA (Madireddi et al., 2000; Sarkar et al., 2007). These reports emphasized the importance of secreted mda- 7 for killing of the neighboring tumor cells; so targeting this protein on tumor cells will improve efficiency and specificity of this anti-tumor gene therapy procedure.

The RGD peptide sequence, due to specific binding to integrin ligands, employed a lot for targeting tumor cells (Craig et al., 2008). The specificity of the RGD4C peptide for $\alpha v \beta 3$ and $\alpha v \beta 1$ integrins (Assa-Munt $N$ et al. 2001), making this peptide a competent candidate for targeting mda-7 to the site of tumors and also for improving the bystander effect.

Limited studies have claimed the usefulness of RGD peptide for targeting mda-7 protein to the specific tumor lines after in vitro production (Xiao et al., 2009; Pei et al., 2012). constructed plasmid expressing RGDmodified mda-7 with novel RGD motif in the middle of mda-7 backbone, then, evaluated its therapeutic efficacy in HepG2 cell line. They demonstrated that expression pattern and apoptotic induction of the new RGD-IL-24 was similar to IL-24 expression plasmid. Theoretically, this kind of design may disrupt true protein folding at the end or some functions albeit they didn't mention it.

We previously constructed 2 new plasmids expressing mda-7 protein which was fused to standard RGD4C (mda7-RGD) and shortened RGD (mda-7-tRGD) peptides, at carboxyl end position to improve the specificity and bystander effect (Khodadad M, In press). In the current study, the major goal was to determine whether RGDmodified mda-7 induce apoptosis better than usual mda-7. Our findings indicated that mda-7 protein exhibited more suppressive effect than two other RGD-modified mda-7, as evaluated by MTT assay, PI/Annexin staining method and BAX gene expression analysis.

The MTT assay on the transfected Hep-G2 cells demonstrated the suppressive role of mda-7 in the Hep-G2 proliferation, compared to the two other RGD-modified proteins. While pmda-7 reduced the viability of transfected cells to near $47 \%$, it remains near $60 \%$ survival for RGD and tRGD-modified mda-7, compared to $77 \%$ viability for pIRES receiving group.

The flow cytometery analysis also was in consistent with MTT results. In this analysis, the percentage of apoptotic events among the cells that received plasmids (only GFP+ cells) was: $37.64 \%$ for mda-7, $18.03 \%$ for mda-7-RGD, $19.92 \%$ for mda-7-tRGD and $12.71 \%$ for IRES. In other words, these data demonstrated that endogenously expressed normal mda-7 plasmid significantly induces apoptosis pathways, compared to other plasmids while no significant difference between others was confirmed. To evaluate bystander effect in culture cells indirectly, flow cytometry results were analyzed either on total cells (both GFP+ and GFP- cells) or those cells received corresponding plasmids (only GFP+ cells). The data analysis in total cell population revealed that apoptosis percentage was: $52.47 \%, 26.17 \%$, $31.86 \%$ and $23.11 \%$ for mda-7, mda-7-RGD, mda-7tRGD and for IRES, respectively. Overall, the comparison between the two sets of analysis revealed that first: only pmda-7 plasmid induces apoptosis at a significant level, in comparison to the two other modified vectors, second: the rate of apoptosis in total cell population was $52.47 \%$ and in gated GFP+ cell was $37.64 \%$ for the cells which received pmda-7, so bystander effect of secreted cytokine is detectable albeit it should be noted that this increment was also measured in pIRES group (23.11 for total cell and $12.71 \%$ for GFP+ cell population), third: new modified versions of mda-7 not only failed to improve apoptotic induction in Hep-G2 cells but even inhibited normal protein to perform usual apoptosis induction. While previous studies demonstrated the helpful role for RGD and NGR peptide in boosting cytokine impact during tumor therapy (Craig et al., 2008) our finding does not support hypothesis considering the improvement of apoptosis induction by tethering RGD peptides to mda-7 . The exact mechanism of RGD-related failure in boosting the apoptosis induction is not clear for us but maybe depends on steric hindrance, possible protein misfolding and unexpected protein interaction during secretion. 
The BAX gene expression analysis also supported flow cytometry data, as expected. In expression analysis, the fold increased in BAX expression was significantly higher for mda-7 and mda-7-tRGD while indicating some troubles associated with apoptotic induction by mda-7-RGD.

Here, to investigate the effect of RGD length, we tested standard RGD-4C motif (CDCRGDCFC amino acid) with truncated RGD (CDGRC) at the end of mda-7 protein. Craig et al, in an effort for targeting cytokine on tumor cells, demonstrated that IFN- $\alpha$-CDGRC protein inhibited tumor growth more significantly than the wild-type IFN- $\alpha$ (Craig et al., 2008) .However, our data demonstrated that tethering RGD to mda-7 alleviated its death inducing property and shortening the length of peptide decreased this inhibitory role.

To elucidate the design failure, the recombinant/wild IL24/mda-7 interaction with its cognate receptor modeled based on IL-20 crystal structure. IL-24 in the ternary complex resembled IL-20 conformation and interaction with three protein interfaces, IL-24/IL-20R site 1-3. After RGD peptide tethering at carboxyl end of IL-24 structure, one motif buried in the center of the ternary complex and disrupted the non-covalent interactions that mediated the formation of ternary complex by IL-24/IL-20R in site 1. It seems that the overall effects of RGD loop was to decrease the affinity of recombinant IL-24 with cognate IL-20R1IL-20R2 receptors when compared to normal protein. This failure inevitably impacted proper attachment of released Il-24 proteins and subsequently weaker signaling responses. Otherwise, this kind of changes may ultimately impacted on mda-7 protein interaction with other protein partners, especially those playing role in cell apoptosis and intracellular trafficking albeit more experiments is demanding. Interestingly, in a similar effort to introduce RGD motif into mda-7 Pei et al (Pei D.S et al, 2012) modified the mda-7 amino acid backbone to obtain RGD from protein original sequence. Even though they didn't mention in those article, those modification doesn't abolish correct attachment potency to its receptors as our modelling analysis revealed well.

In conclusion, aaccording to the results, mda-7 overexpression triggers apoptotic pathways inside Hep-G2 tumor cell but fusion with RGD4C or truncated RGD forms, decreased this propensity by an uncharacterized mechanism. The tethering of targeting peptide to mda-7 or other cytokine will not always get better response, and different kinds of design should be assessed to achieve more sustained results. The 3D modeling results highlighted that while mda-7 structure left intact after RGD peptides adding, exact attachment to cognate receptor abolished due to new conformations. This change may also disrupt other protein interaction responsible for apoptosis induction by mda- 7 .

\section{Acknowledgements}

The authors would like to appreciate Dr. Abbas Ghaderi, Head of Shiraz Institute of Cancer research (ICR) and Dr. Maryam Ardebili, manager of Gastroenterohepatology research center for all cooperation and supports. We also thank Fatemeh Rezaei, Maryam Nejabat, Mastaneh Zeraatian, Saeed Amirzadeh and Maryam Talaei for their laboratory technical support. This work was supported by grant number 91-01-13-4647 from Shiraz University of Medical Sciences, Shiraz, Iran. Finally the authors wish to thank Dr. Hassan Khajehei, for copy editing of the manuscript.

\section{References}

Assa-Munt N, Jia X, Laakkonen P, et al (2001). Solution structures and integrin binding activities of an RGD peptide with two isomers. Biochemist, 40, 2373-8.

Bazan-Peregrino M, Seymour L, Harris A (2007). Gene therapy targeting to tumor endothelium. Cancer gene therap, 14, 117-27.

Caudell EG, Mumm JB, Poindexter N, et al (2002). The protein product of the tumor suppressor gene, melanoma differentiation-associated gene 7 , exhibits immunostimulatory activity and is designated IL-24. $J$ Immunol, 168, 6041-6.

Chada S, Bocangel D, Pataer A, et al (2007). MDA-7/IL-24 as a multi-modality therapy for cancer. In 'Gene Therapy for Cancer', Eds Springer, 413-33

Chada S, Mhashilkar AM, Ramesh R, et al (2004). Bystander activity of Ad-mda7: human MDA-7 protein kills melanoma cells via an IL-20 receptor-dependent but STAT3independent mechanism. Molecular Therap, 10, 1085-95.

Craig R, Cutrera J, Zhu S, et al (2008). Administering Plasmid DNA Encoding Tumor Vessel-anchored IFN- $\alpha$ for Localizing Gene Product Within or Into Tumors. Molecular Therap, 16, 901-6.

DeLano WL (2002). The PyMOL molecular graphics system.

Fisher PB (2005). Is mda-7/IL-24 a "magic bullet" for cancer? Cancer Res, 65, 10128-38.

Fisher PB, Gopalkrishnan RV, Chada S, et al (2003). mda-7/ IL-24, a novel cancer selective apoptosis inducing cytokine gene: from the laboratory into the clinic. Cancer biol Therap, 2, 22-36.

Gupta P, Su Z-z, Lebedeva IV, et al (2006). mda-7/IL-24: multifunctional cancer-specific apoptosis-inducing cytokine. Pharmacol Therapeut, 111, 596-628.

Hersel U, Dahmen C, Kessler H (2003). RGD modified polymers: biomaterials for stimulated cell adhesion and beyond. Biomaterials, 24, 4385-415.

Holig P, Bach M, Volkel T, et al (2004). Novel RGD lipopeptides for the targeting of liposomes to integrin-expressing endothelial and melanoma cells. Protein Engineer Design Select, 17, 433-41.

Huang EY, Madireddi MT, Gopalkrishnan RV, et al (2001). Genomic structure, chromosomal localization and expression profile of a novel melanoma differentiation associated (mda-7) gene with cancer specific growth suppressing and apoptosis inducing properties. Oncogene, 20, 7051-63.

Jiang H, Lin JJ, Su Z, et al (1995). Subtraction hybridization identifies a novel melanoma differentiation associated gene, mda-7, modulated during human melanoma differentiation, growth and progression. Oncogene, 11, 2477-86.

Jiang H, Su Z-Z, Lin JJ, et al (1996). The melanoma differentiation associated gene mda-7 suppresses cancer cell growth. Proc Natl Acad Sci U S A, 93, 9160-5.

Khodadad M HS-Y, Shenavar F, Erfani N, Bina S, Ahmadian S, Fattahi M-R, Hajhosseini R (In press). Construction of expressing vectors including mda-7 gene beside the RGD sequences for better tumor targeting. Iran J Basic Res. [Epub ahead of print] 


\section{Samaneh Bina et al}

Kunz S, Wolk K, Witte E, et al (2006). Interleukin (IL)-19, IL-20 and IL-24 are produced by and act on keratinocytes and are distinct from classical ILs. Experimental Dermatol, 15, 991-1004.

Lebedeva IV, Emdad L, Su Z-Z, et al (2007). mda-7/IL-24, novel anticancer cytokine: focus on bystander antitumor, radiosensitization and antiangiogenic properties and overview of the phase I clinical experience (Review). Int $J$ Oncol, 31, 985-1007.

Lebedeva IV, Su ZZ, Sarkar D, et al (2003). Melanoma differentiation associated gene-7, mda-7/interleukin-24, induces apoptosis in prostate cancer cells by promoting mitochondrial dysfunction and inducing reactive oxygen species. Cancer Res, 63, 8138-44.

Logsdon NJ, Deshpande A, Harris BD, et al (2012). Structural basis for receptor sharing and activation by interleukin-20 receptor-2 (IL-20R2) binding cytokines. Proceedings National Acad Sci, 109, 12704-9.

Madireddi MT, Dent P, Fisher PB (2000). Regulation of mda-7 gene expression during human melanoma differentiation. Oncogene, 19, 1362-8.

Madireddi MT, Su Z-Z, Young CS, et al (2002). Mda-7, a novel melanoma differentiation associated gene with promise for cancer gene therapy. In 'Cancer Gene Therapy', $E d s$ Springer, 239-61

Marchand-Brynaert J, Detrait E, Noiset O, et al (1999). Biological evaluation of RGD peptidomimetics, designed for the covalent derivatization of cell culture substrata, as potential promotors of cellular adhesion. Biomaterials, $\mathbf{2 0}$, 1773-82.

McGuffin LJ, Bryson K, Jones DT (2000). The PSIPRED protein structure prediction server. Bioinformatics, 16, 404-5.

McLane MA, Vijay-Kumar S, Marcinkiewicz C, et al (1996). Importance of the structure of the RGD-containing loop in the disintegrins echistatin and eristostatin for recognition of $\alpha \mathrm{IIb} \beta 3$ and $\alpha v \beta 3$ integrins. FEBS letters, 391, 139-43.

Pei D-S, Yang Z-X, Zhang B-F, et al (2012). Enhanced apoptosisinducing function of MDA-7/IL-24 RGD mutant via the increased adhesion to tumor cells. J Int Cytokine Res, 32, 66-73.

Sarkar D, Lebedeva IV, Gupta P, et al (2007). Melanoma differentiation associated gene-7 (mda-7)/IL-24: a'magic bullet'for cancer therapy?

Sarkar D, Su Z-Z, Lebedeva IV, et al (2002). mda-7 (IL-24) Mediates selective apoptosis in human melanoma cells by inducing the coordinated overexpression of the GADD family of genes by means of p38 MAPK. Proc Natl Acad Sci U S A, 99, 10054-9.

Sauane M, Gopalkrishnan RV, Lebedeva I, et al (2003). Mda-7/ IL-24 induces apoptosis of diverse cancer cell lines through JAK/STAT-independent pathways. J Cellular Physiol, 196, 334-45.

Schwede T, Kopp J, Guex N, et al (2003). SWISS-MODEL: an automated protein homology-modeling server. Nucleic Acids Res, 31, 3381-5.

Su Z-Z, Lebedeva IV, Sarkar D, et al (2003). Melanoma differentiation associated gene-7, mda-7/IL-24, selectively induces growth suppression, apoptosis and radiosensitization in malignant gliomas in a p53-independent manner. Oncogene, 22, 1164-80.

Su Z, Emdad L, Sauane M, et al (2005). Unique aspects of mda-7/IL-24 antitumor bystander activity: establishing a role for secretion of MDA-7/IL-24 protein by normal cells. Oncogene, 24, 7552-66.

Whitaker EL, Filippov VA, Duerksen-Hughes PJ (2012). Interleukin 24: mechanisms and therapeutic potential of an anti-cancer gene. Cytokine Growth Factor Rev, 23, 323-31.
Wu S, Zhang Y (2007). LOMETS: a local meta-threadingserver for protein structure prediction. Nucleic Acids Res, 35, 3375-82.

Xiao B, Li W, Yang J, et al (2009). RGD-IL-24, a novel tumortargeted fusion cytokine: expression, purification and functional evaluation. Molecular Biotechnol, 41, 138-44.

Xiong J-P, Stehle T, Zhang R, et al (2002). Crystal structure of the extracellular segment of integrin $\alpha \mathrm{V} \beta 3$ in complex with an Arg-Gly-Asp ligand. Science, 296, 151-5.

Yamamoto M, Curiel DT (2005). Cancer gene therapy. Technol Cancer Res Treat, 4, 315-30.

Zhang Y (2008). I-TASSER server for protein 3D structure prediction. BMC Bioinformat, 9, 40. 